\title{
Anti-EGFR mAb cetuximab therapy increases $T$ cell receptor (TCR) diversity in the peripheral blood and focuses TCR richness in the tumor microenvironment
}

\author{
Benjamin A Kansy ${ }^{1 *}$, Yan Lin² ${ }^{2}$ Fei Ding ${ }^{2}$, Sandra Poveda Gibson ${ }^{3}$, Hyun-Bae Jie ${ }^{3}$, Robert L Ferris ${ }^{3}$ \\ From 30th Annual Meeting and Associated Programs of the Society for Immunotherapy of Cancer (SITC 2015) \\ National Harbor, MD, USA. 4-8 November 2015
}

\section{Introduction}

$\mathrm{T}$ cell receptor (TCR) recognition of tumor antigen is essential for effective antitumor immunity. The immune system `s ability to respond to a broad spectrum of antigens requires a sophisticated selection of various TCR. So far, little is known about the role of TCR richness and clonality in the immune response to the EGFR-specific $\mathrm{mAb}$, cetuximab. Therefore, we investigated differences in TCR sequences between $\mathrm{HPV}^{+}$and $\mathrm{HPV}^{-}$patients, as well as differences in sequence characteristics between $\mathrm{T}$ cells of peripheral blood mononuclear cells (PBMC) and tumor infiltrating lymphocytes (TIL). Additionally, we were able to investigate the TCR richness and clonality in samples pre- and post-treatment in a clinical single agent cetuximab trial.

\section{Material and methods}

TCR genotyping was performed and quantified by Adaptive Technologies, Inc (Seattle, WA). Richness (S) was defined as total number of unique productive sequences. The Shannon Index is defined as $\mathrm{H}=-\log \sum \mathrm{p}_{\mathrm{i}}\left(1-\mathrm{p}_{\mathrm{i}}\right)$, $\mathrm{p}_{\mathrm{i}}$ is the proportion of sequence $i$ relative to the total sequences. The clonality is defined as $1-H / \log (\mathrm{S})$. The maximum available number of samples from the same patient was 4 , containing pre- and post-treatment PBMC and pre- and post-treatment TIL. Paired comparisons (pre vs. post) were done using the Wilcoxon Signed Rank tests. Comparisons between two independent groups (i.e. $\mathrm{HPV}^{+}$vs. $\mathrm{HPV}^{-}$, or responders vs. non-responders) were accomplished with Wilcoxon Rank Sum tests.

'Department of Otorhinolaryngology, University Hospital Essen, Germany;

University of Pittsburgh Cancer Institute, Pittsburgh, PA, USA

Full list of author information is available at the end of the article

\section{Results}

Using a cohort of neoadjuvant, single-agent cetuximab treated HNSCC patients, 56 samples were analyzed for global TCR diversity. $\mathrm{HPV}^{+}$and $\mathrm{HPV}^{-}$patients did not significantly differ in clonality and richness pre- and post-treatment in either PBMC or TIL. However, cetuximab therapy significantly increased the richness of unique sequences in PBMC ( $\mathrm{p}=0.0002)$. Most importantly, the responder group had a higher increase of richness post-treatment in PBMC. The difference reached a $\mathrm{p}$-value of 0.03 post-treatment. On the contrary, this increase was not observed in TIL, which appeared to be more focused after cetuximab therapy.

\section{Discussion}

TCR clonality and richness for PBMC and TIL seem to be independent from the patient's HPV status in HNSCC. Intriguingly, an increase in richness of TCR sequences in the periphery (PBMC) but a focusing in TIL was associated with an improved treatment response, suggesting an influence of peripheral quantity on intratumoral TCR quality.

\section{Authors' details \\ ${ }^{1}$ Department of Otorhinolaryngology, University Hospital Essen, Germany; University of Pittsburgh Cancer Institute, Pittsburgh, PA, USA. ${ }^{2}$ Biostatistics Facility, University of Pittsburgh Cancer Institute, Pittsburgh, PA, USA. ${ }^{3}$ University of Pittsburgh Cancer Institute, Pittsburgh, PA, USA.}

Published: 4 November 2015

\section{doi:10.1186/2051-1426-3-S2-P73}

Cite this article as: Kansy et al:: Anti-EGFR mAb cetuximab therapy increases $T$ cell receptor (TCR) diversity in the peripheral blood and focuses TCR richness in the tumor microenvironment. Journal for ImmunoTherapy of Cancer 2015 3(Suppl 2):P73. 